\title{
Contribution empirique à l'étude de la propagation de la houle à l'intérieur des digues
}

\author{
An empirical contribution to the study of swell \\ propagation in a breakwater
}

\author{
A. Hakimi $\quad$ A. Diouri M. Kaissoun \\ Laboratoire public d'essais et d'études \\ Casablanca, Maroc
}

Si les écoulements d'eau dans un barrage en terre commencent à être de mieux en mieux cernés, ceux à travers une digue en mer restent mal connus et ceci principalement à cause, d'une part des dimensions importantes des matériaux qui constituent le noyau de la digue, et d'autre part de la houle qui sollicite l'ouvrage et confère au phénomène un caractère dynamique, et donc non statique comme celui des barrages. C'est pour pallier à cette méconnaissance que l'Administration marocaine de l'Equipement a fait appel au Laboratoire public d'essais et d'études pour qu'il propose et mette en exécution une méthode expérimentale permettant de mieux approcher le phénomène.

Le problème s'est posé concrètement lors de la construction du port de Jorf Lasfar, et celà à l'occasion de l'étude de stabilité des ouvrages de couronnement, et surtout quand il s'est agi de dimensionner la dalle de circulation compte tenu des efforts de sous-pressions. Or ces forces dépendent de l'agitation qui règne au pied de la digue et de la manière dont elle se propage et s'amortit dans le noyau. C'était là le premier but de l'opération, mais assez vite on a débouché sur d'autres questions, auxquelles il fallait répondre :

1) Détermination des lignes d'eau et de courant et leur variation en fonction de l'agitation au pied de la digue.

2) Détermination des pressions interstitielles pour les calculs de stabilité dynamique.

3) La variation dans le temps et sur plusieurs années de la perméabilité du noyau de la digue.

Nous tenons à préciser dès maintenant que tous ces buts n'ont pas été atteints, mais nous avons quand même abouti à une méthode expérimentale qui permet de répondre aux questions posées si elle est améliorée par les critiques et les conseils des spécialistes dans le domaine.

Nous donnons ci-après la description du dispositif de mesure, le résumé des résultats obtenus, et partant des difficultés rencontrées, nous donnons en conclusion les améliorations que nous prévoyons d'apporter au système de mesure pour aboutir à des meilleurs résultats.

\section{Dispositif de mesure}

La digue de Jorf Lasfar est du type classique : à talus, carapace extérieure en tétrapodes de $20 \mathrm{~m}^{3}$, sous couche en enrochements, avec noyau central en tout-venant ( 0 à $1500 \mathrm{~kg}$ ), dalle de couronnement et mur de garde en béton. La figure 1 montre un profil type de la digue.

Deux profils transversaux ont été muni de 4 piézomètres verticaux de $11 \mathrm{~m}$ de profondeur à partir du niveau de la chaussée définitive. La base des piézomètres est sous le zéro hydrographique, à $3 \mathrm{~m}$ pour le $\mathrm{PM} 800$, et à $2 \mathrm{~m}$ pour le PM 1800.

Les piézomètres ont été introduits dans des forages réalisés préalablement. Ils sont constitués par des tubes de gaz filetés, crépinés sur les $5 \mathrm{~m}$ inférieurs, avec le fond soudé pour éviter la montée de vase dans les piézomètres. Le crépinage offre une section de passage à l'eau de $8 \%$, par des fentes de $1,5 \mathrm{~mm}$. Un massif de gravier filtrant a été disposé dans l'espace annulaire entre le forage et le piézomètre; le diamètre intérieur de celui-ci est de $67 \mathrm{~mm}$.

Les quatres piézomètres de chaque profil sont distants les uns des autres d'à peu près $3,50 \mathrm{~m}$ (la largeur de la chaussée est de $12 \mathrm{~m}$ ).

Afin de ne pas perturber l'écoulement réel dans la digue, le diamètre des piézomètres a été choisi le plus petit et le crépinage le plus large possible, compte tenu de la possibilité d'introduction des capteurs et de la résistance mécanique des tubes.

D'autre part, vu la distance entre les piézomètres extrêmes d'un même profil, environ $10 \mathrm{~m}$, et leur disposition à partir de la chaussée, les mesures ont intéressé uniquement la partie centrale du noyau de la digue. 


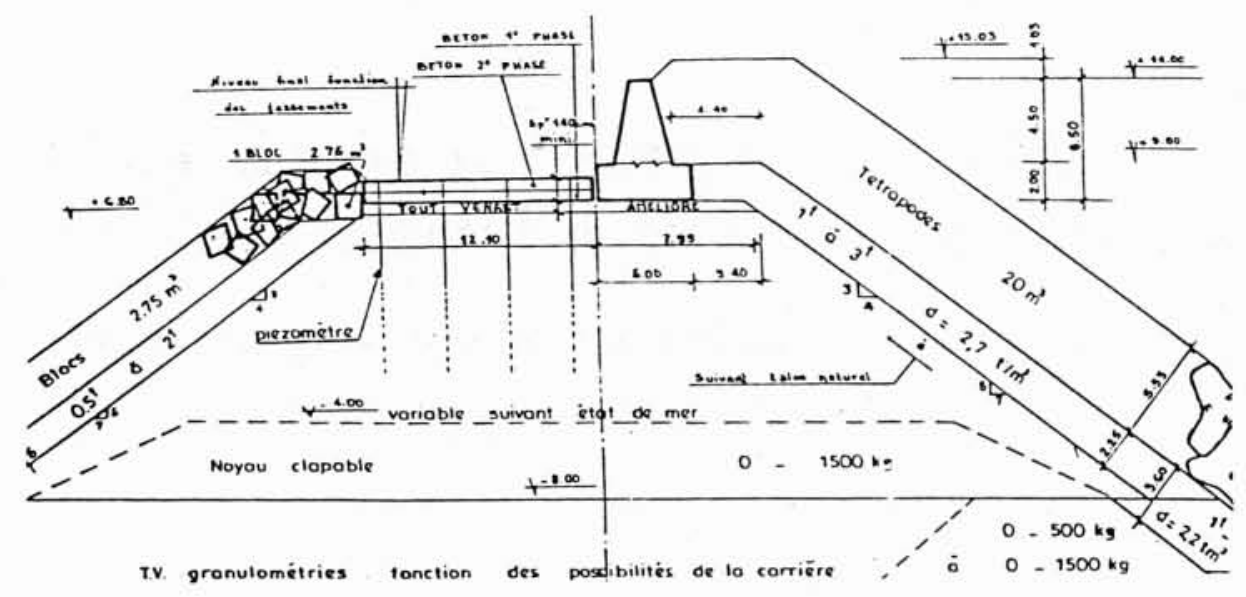

Figure 1

Le matériel de mesure utilisé était le suivant :

- des capteurs de pression à jauge de contrainte;

- un enregistreur graphique à 2 pistes d'enregistrement;

- des câbles blindés et étanches reliant les sondes de mesures au système électrique de stabilisation et d'enregistrement.

Les capteurs de pression ont été introduits à proximité du fond des piézomètres qui se trouve à 2 ou $3 \mathrm{~m}$ sous le zéro hydrographique; ils ont été par conséquent, durant toute la durée d'enregistrement, complètement noyés. Ainsi, la pression qu'ils ont pu enregistrer peut être confondue avec la pression hydrostatique.

L'enregistrement des niveaux d'eau dans le noyau de la digue a été accompagné par celui de la houle au large fait par la bouée Datawell du chantier qui transmet par radio l'information à un ordinateur. Celui-ci traite l'information sur des périodes de 15 minutes et donne les caractéristiques de la houle correspondante: temps de l'enregistrement, hauteur maximale $H_{\max }$, hauteur significative $H_{s}$, période moyenne $T$, période $T_{H 1 / 3}$.

Notons que la houle, quand elle s'approche de la digue, subit un certain nombre de modifications dans la direction, l'amplitude, la longueur d'onde, la célérité et non la période qui, en principe, reste constante. Elles sont dues à sa réfraction, réflexion, déferlement. Le concours de tous ces phénomènes donne des houles à proximité de la digue très complexes à prédire par de simples considérations théoriques. Et pourtant, c'est à partir de ces clapotis qu'il faudrait voir les capacités d'amortissement et de drainage de la digue. Il aurait été, par conséquent, fort intéressant d'effectuer un enregistrement de la houle au pied de la digue et au PM de mesure en même temps que celui du niveau d'eau dans le noyau.

Ceci dit, les mesures ont été effectuées pratiquement de la façon suivante :

- enregistrements de 15 à 20 minutes;

- enregistrement simultané de deux piézomètres à la fois (l'enregistreur utilisé disposant de deux pistes d'enregistrements);

- en numérotant les piézomètres de 1 à 4 en allant du côté large au côté port, les paires de piézomètres suivantes ont été enregistrées : 1 et 2,1 et 3,1 et 4,2 et 3,2 et 4 , 3 et 4 ;

- pour chaque enregistrement, on a noté exactement l'heure et la minute du début et de la fin de l'enregistrement. Celà est nécessaire pour comparer avec la houle au large enregistrée par la bouée, et pour déterminer les hauteurs de la marée correspondante. Il a été également noté, pour chaque enregistrement, les profondeurs des deux capteurs à partir du niveau supérieur de la dalle.

\section{Dépouillement et résultats}

Comme on ne disposait pas de mesure de houle au pied de la digue, on n'avait comme houle de référence que celleenregistrée par la bouée Datawell au large de la digue et l'on ne pouvait pas faire un dépouillement vague par vague. On a donc travaillé sur un enregistrement complet de 15 à 20 minutes.

Pour chaque enregistrement de la pression dans un piézomètre, nous avons déterminé : l'amplitude maximale, l'amplitude significative et la période moyenne. La méthode du dépouillement utilisée est celle du "zéro up crossing ".

Or, pour cela il est nécessaire de déterminer le niveau de référence des fluctuations ou le niveau moyen à l'intérieur de la digue.

Il est à noter que ce niveau moyen à l'intérieur de la digue pourrait être pris, pour une première approximation égal au niveau de repos dans le bassin du port c'est-à-dire au niveau de la marée. Cela est d'autant plus vrai que le tout venant de la digue est plus drainant. 
Il est bien évident que si l'on avait disposé d'une acquisition numérique des mesures, on aurait pu faire une analyse plus précise et surtout basée sur une comparaison des énergies, ce qui aurait été beaucoup plus représentatif pour les capacités d'amortissement de la digue.

Cependant, si l'on ne pouvait pas faire une comparaison vague par vague entre l'enregistrement de la bouée Datawell et celui des piézomètres, on pouvait le faire entre deux enregistrements d'un piézomètre à un autre, la correspondance était assez visible.

En ce qui concerne les mesures, elles ont été effectuées lors des trois campagnes : - mars 1981, - décembre 1981, et - mars 1983 .

Nous avons classé les résultats d'une part par ordre chronologique pour avoir une idée sur la variation des caractéristiques du noyau de la digue au cours du temps et d'autre part on a classé les résultats par amplitudes croissantes de la houle incidente afin de pouvoir tirer des coefficients d'amortissement pour chaque gamme d'amplitude.

\section{Campagne $n^{\circ} 1$}

\begin{tabular}{|c|c|c|c|c|c|c|}
\hline \multirow[t]{2}{*}{ Essais $n^{\circ}$} & \multirow{2}{*}{$\begin{array}{l}\text { Marée } \\
H_{\max }(\mathrm{m})\end{array}$} & \multirow{2}{*}{$\begin{array}{l}\text { Houle } \\
H_{s}(\mathrm{~m})\end{array}$} & \multicolumn{4}{|c|}{\begin{tabular}{|ll} 
Fluctuation maximale dans le \\
piézomètre $n^{\circ}$ & $(\mathrm{m})$
\end{tabular}} \\
\hline & & & $h_{1}$ & $h_{2}$ & $h_{3}$ & $h_{4}$ \\
\hline 30 & 1,6 & 7,08 & 0,88 & 1,10 & - & - \\
\hline 28 & 1,80 & 5,93 & 1,375 & - & - & 0,77 \\
\hline 31 & 1,42 & 5,61 & - & 1,485 & - & 0,99 \\
\hline 34 & 1,25 & 5,33 & - & - & 0,66 & 0,77 \\
\hline 35 & 1,20 & 5,33 & - & 1,10 & 0,715 & - \\
\hline 29 & 1,65 & 4,65 & 1,32 & - & 1,76 & - \\
\hline 45 & 1 & 4,47 & 0,88 & 1,10 & - & 0,88 \\
\hline 1 & 1,5 & 4,12 & 0,88 & - & - & 0,555 \\
\hline 42 & 1,60 & 3,93 & 0,88 & - & - & 0,99 \\
\hline 44 & 1,1 & 3,56 & 1,155 & 1,21 & - & - \\
\hline 41 & 1,80 & 3,51 & 1,21 & - & - & 1,32 \\
\hline 57 & 3,80 & 3,22 & - & - & 0,385 & 0,50 \\
\hline 43 & 1,4 & 2,97 & 1,21 & - & 1,21 & - \\
\hline 56 & 3,85 & 2,97 & - & 0,88 & 0,88 & - \\
\hline 12 & 1,45 & 2,91 & 0,66 & - & - & - \\
\hline 13 & 1,35 & 2,91 & - & 1,005 & - & - \\
\hline 18 & 1,15 & 2,87 & - & - & 1,032 & - \\
\hline 53 & 3,7 & 2,73 & 0,99 & - & 0,88 & - \\
\hline 54 & 3,8 & 2,73 & 0,99 & 0,77 & - & - \\
\hline 55 & 3,85 & 2,73 & - & 0,66 & - & 0,66 \\
\hline 10 & 3,10 & 254 & 121 & - & 1.10 & - \\
\hline 15 & 1,35 & 2,54 & - & - & - & 0,744 \\
\hline 17 & 1,32 & 2,37 & - & 1,089 & - & - \\
\hline 52 & 3,65 & 2,35 & 1,155 & - & - & 0,90 \\
\hline 5 & 3,10 & 2,16 & 1,21 & 1,65 & - & - \\
\hline 6 & 3,10 & 2,09 & 1,21 & - & 1,21 & - \\
\hline 8 & 3,10 & 1,97 & - & 1,10 & - & 0,44 \\
\hline
\end{tabular}

Campagne $n^{\circ}$ 2. - PM $1800-$

\begin{tabular}{|c|c|c|c|c|c|c|}
\hline \multirow[t]{2}{*}{ Essais $n^{\circ}$} & \multicolumn{2}{|c|}{ Houle } & \multicolumn{4}{|c|}{$\begin{array}{l}\text { Fluctuation maximale dans le } \\
\text { pièzomètre } \mathrm{n}^{\circ}\end{array}$} \\
\hline & $H_{\max }(\mathrm{m})$ & $\mathrm{H}_{s}$ & 1 & 2 & 3 & 4 \\
\hline 17 & 9,2 & 4,65 & - & 1,08 & - & 0,53 \\
\hline 15 & 8,63 & 4,97 & 0,92 & - & - & 0,53 \\
\hline 8 & 8,05 & 4,92 & 0,92 & - & - & - \\
\hline 18 & 7,64 & 5,18 & 0,53 & 0,92 & - & - \\
\hline 13 & 7,63 & 4,75 & - & 1,20 & 0,62 & - \\
\hline 14 & 7,63 & 5 & - & - & 0,77 & 0,40 \\
\hline 9 & 7,61 & 4,92 & - & 1,73 & 0,62 & - \\
\hline 11 & 7,52 & 4,83 & 1,08 & - & - & - \\
\hline 19 & 7,24 & 5,17 & 0,53 & - & - & 0,77 \\
\hline 25 & 7,01 & 4,11 & - & 0,92 & - & - \\
\hline 10 & 9,96 & 4,62 & - & - & - & 0,77 \\
\hline 12 & 6,80 & 4,38 & - & 1,20 & - & 6 \\
\hline 26 & 6,61 & 3,88 & - & 1,06 & - & 0,46 \\
\hline 22 & 6.47 & 4,28 & - & - & 0,93 & 0,46 \\
\hline 24 & 6,23 & 4,41 & - & 0,92 & - & - \\
\hline 23 & 5,97 & 4,25 & - & - & 0,77 & 1,20 \\
\hline 6 & 4,15 & 2,48 & - & 1,06 & - & 0,46 \\
\hline 1 & 3,89 & 2,31 & 0,53 & 1,77 & - & - \\
\hline 2 & 3,89 & 2,31 & - & 0,77 & 0,53 & - \\
\hline 3 & 3,89 & 2,31 & - & - & 0,53 & 0,77 \\
\hline 4 & 3,86 & 2,37 & 1,06 & - & - & 0,77 \\
\hline 5 & 3,86 & 2,37 & 1,20 & - & 0,62 & - \\
\hline 7 & 3,66 & 2,56 & - & 0,93 & 0,92 & - \\
\hline
\end{tabular}

Campagne $n^{\circ}$ 3. - PM 1800 -

Mesures du 23-24 mars 1983

\begin{tabular}{|c|c|c|c|ccc|}
\hline Essais $n^{\circ}$ & Marée & Houle & \multicolumn{5}{|c|}{$\begin{array}{c}\text { Fluctuation maximale dans le } \\
\text { piézomètre } \mathrm{n}^{\circ}\end{array}$} \\
& & & \multicolumn{5}{c}{$\begin{array}{c}\text { m) } \\
\end{array}$} & $H_{\max }(\mathrm{m})$ & $H_{s}$ & 1 & 2 & 3 & 4 \\
\hline 1 & 3,50 & 2,41 & 0,90 & 0,57 & - & - \\
2 & 3,50 & 2,41 & 1,10 & - & 1,00 & - \\
3 & 4,04 & 2,69 & 1,10 & - & - & 1,00 \\
4 & 3,91 & 2,36 & - & 0,55 & 1,00 & - \\
5 & 3,93 & 2,34 & - & 0,70 & 0,67 & - \\
& & & & & & \\
6 & 3,94 & 2,32 & - & - & 0,90 & 0,86 \\
7 & 2,80 & 1,85 & - & 0,45 & - & 0,71 \\
8 & 3,10 & 2,13 & 1,00 & 0,55 & - & - \\
9 & 3,71 & 2,27 & 0,86 & - & 0,78 & - \\
10 & 3,44 & 2,12 & 0,78 &,- 08 & - & 0,56 \\
& & & & & & \\
11 & 3,11 & 2,09 & - & 0,57 & 0,67 & - \\
12 & 3,05 & 2,02 & - & 0,50 & - & 0,50 \\
13 & 3,16 & 2,02 & - & - & 0,86 & 0,56 \\
\hline
\end{tabular}

Mesures du 31 mars 1983

\begin{tabular}{|c|c|c|c|c|}
\hline Essais $\mathrm{n}^{\circ}$ & Piézo 1 & 2 & 3 & 4 \\
\hline 1 & 0,40 & 0,18 & - & - \\
2 & 0,51 & - & 0,18 & - \\
3 & 0,40 & - & - & 0,37 \\
4 & - & 0,37 & $\overline{-}$ & 0,30 \\
5 & - & - & 0,27 & 0,30 \\
6 & - & 0,40 & 0,18 & - \\
\hline
\end{tabular}




\section{Tableaux des coefficients d'amortissements}

Les coefficients moyens d'amortissement sont définis tel que :

$$
\mathrm{Am}_{\mathrm{i}}=H_{\max } \text { bouée } / H_{\max } \text { piézo } \mathrm{n}^{\circ} i
$$

\begin{tabular}{|c|c|c|}
\hline Campagne & $\begin{array}{c}\text { PM 1800 } \\
\text { Am }_{1}\end{array}$ & $\begin{array}{c}\text { PM 800 } \\
\text { Am, }\end{array}$ \\
\hline 1 & 3,27 & 2,87 \\
\hline 2 & 7,49 & - \\
\hline 3 & 3,71 & 2,99 \\
\hline
\end{tabular}

Le détail pour chaque campagne est :

\section{Campagne $n^{\circ} 1$}

\begin{tabular}{|c|c|c|c|c|}
\hline & $A m_{1}$ & $\mathrm{Am}_{2}$ & $\mathrm{Am}_{3}$ & $\mathrm{Am}_{4}$ \\
\hline $5 \mathrm{~m}<H_{\max }<7 \mathrm{~m}$ & 5,76 & 4,89 & 7,75 & 6,66 \\
\hline $3 \mathrm{~m}<H_{\max }<5 \mathrm{~m}$ & 3,83 & 3,47 & 3,66 & 4,54 \\
\hline $2 \mathrm{~m}<H_{\max }<3 \mathrm{~m}$ & 2,37 & 2,49 & 2,56 & 3,49 \\
\hline
\end{tabular}

\section{Campagne $n^{\circ} 2$}

\begin{tabular}{|c|c|c|r|r|}
\hline & $A m_{1}$ & $\mathrm{Am}_{2}$ & $\mathrm{Am}_{3}$ & $\mathrm{Am}_{4}$ \\
\hline $5 \mathrm{~m}<H_{\max }<7 \mathrm{~m}$ & 9,8 & 6,68 & 11,37 & 14,66 \\
\hline $3 \mathrm{~m}<H_{\max }<5 \mathrm{~m}$ & - & 6,17 & 7,31 & 13,76 \\
\hline $2 \mathrm{~m}<H_{\max }<3 \mathrm{~m}$ & 4,16 & 4,61 & 5,88 & 5,95 \\
\hline
\end{tabular}

\section{Campagne $n^{\circ} 3$}

\begin{tabular}{|c|c|c|c|c|}
\hline & $\mathrm{Am}_{1}$ & $\mathrm{Am}_{2}$ & $\mathrm{Am}_{3}$ & $\mathrm{Am}_{4}$ \\
\hline $3 \mathrm{~m}<H_{\max }<5 \mathrm{~m}$ & 3,72 & 6 & 4,15 & 5,06 \\
\hline
\end{tabular}

N.B. : Il faut noter que le piézomètre 2 était particulièrement bouché lors des campagnes 2 et 3 .

L'examen des tableaux ci-dessus et de l'ensemble des résultats que nous n'avons pu reproduire ici nous permet de tirer les conclusions suivantes :

- l'amortissement et d'autant plus grand que la houle incidente est plus importante;

- la plus grande partie de l'énergie de la houle est dissipée dans la carapace;

- la digue devient de plus en plus imperméable, ce qui s'explique par le tassement progressif et le non départ des fines;

- l'amplitude des grandes fluctuations dans la digue est de $1,7 \mathrm{~m}$ pour des houles extérieures de $9 \mathrm{~m}$;

- l'amortissement de la houle est plus important au PM 1800 qu'au PM 800;

- les forces de sous-pressions sont assez faibles pour les houles inférieures à $9 \mathrm{~m}$;

- la période des fluctuations dans la digue est pratiquement le double de la période de la houle enregistrée par la bouée Datawell.
Remarques sur les mesures déjà effectuées et perspectives d'avenir

1) Durant les mesures par temps de tempête, l'équipe chargée de celles-ci, n'a cessé d'être submergée par les franchissements par-dessus le mur de garde. Et vu qu'elle ne disposait que d'un abri de fortune elle a été à plusieurs reprises envahie par l'eau qui noyait les appareils et les mettait hors d'état de fonctionnement pendant une bonne partie du temps consacrée aux mesures.

Ces conditions difficiles ont beaucoup nuit au déroulement des mesures : surtout au moment où le franchissement survenait à l'endroit du PM de mesure. Il faudrait noter que c'est au moment du franchissement, que les sous-pressions éventuelles peuvent survenir.

Pour résoudre ce problème posé par l'enregistrement des mesures sur la jetée même, on a pensé à envoyer l'enregistrement assez loin du profil de mesure dans un local protégé se trouvant en dehors de l'atteinte des franchissements.

Cela nécessite une longueur du câble de liaison plus importante qui entraîne une atténuation du signal; d'où l'obligation d'adopter un amplificateur, lequel amplificateur nécessite lui-même une protection contre les eaux franchissantes.

On pourrait résoudre le problème autrement en disposant des capteurs de pression qui donnent un signal de sortie en fréquence et non en tension, le signal en fréquence ne subissant pas d'atténuation en fonction de la longueur du câble de raccordement.

2) Un certain nombre de piézomètres ont été envasés, ce qui a contribué à amortir les fluctuations de niveau dans le piézomètre par les pertes de charges supplémentaires introduites ainsi que par la diminution de la section offerte au passage de l'eau.

Nous avons vu donc la nécessité d'entretenir régulièrement les piézomètres en y injectant de l'eau sous-pression.

3) Nous avons constaté pour les trois campagnes de mesures l'apparente indépendance entre les caractéristiques de la houle au large et celle des fluctuations de niveau à l'intérieur de la digue. En effet, il nous a été pratiquement impossible d'établir des corrélations enre les deux phénomènes qui sont, en principe, en étroite dépendance. Cela peut être dû à plusieurs causes :

- la difficulté de synchronisation entre la houle au large et les fluctuations de niveau dans la digue. Effectivement, pour avoir une bonne synchronisation entre les deux phénomènes il faudrait caler correctement la montre du chantier (sur laquelle on se base pour repérer les enregistrements des fluctuations dans le temps) sur l'heure indiquée par l'ordinateur relié à la bouée d'enregistrement de la houle au large; d'autre part, il faudrait espérer de telle sorte que les périodes d'enregistrements de la houle au large (période de $15 \mathrm{mn}$ ou une heure suivant l'importance de la valeur de la hauteur significative) coïncident avec celles des enregistrements des fluctuations ou au moins que les premières soient contenues dans les dernières; 
- en outre, pour la synchronisation, il faudrait estimer le temps de propagation des ondes entre la bouée et la digue. Ce temps dépend de la célérité de la houle; et donc de la longueur d'onde et de la bathymétrie des fonds parcourus.

Ainsi, en regard de toutes ces complications pour obtenir une bonne synchronisation, il faudrait chercher les corrélations entre les fluctuations de niveau dans la digue et le clapotis partiel au pied de celle-ci et non point la houle au large;

4) L'enregistreur que nous avons utilisé disposait de deux pistes alors que nous avions 4 piézomètres, celà a entrainé des difficultés dans le dépouillement des résultats.

5) Le signal qui doit être perçu par les capteurs est certainement perturbé par le fait de leur emplacement à l'intérieur des tubes crépinés. Ces derniers introduisent des pertes de charge diminuant les amplitudes et augmentant les périodes. Par conséquent, il est plus judicieux de mettre les capteurs en contact direct avec le tout venant du noyau. Mais, des problèmes d'accès, de mise en place, d'isolation des capteurs et de liaison avec l'enregistreur se posent.

Toutefois, pour garder une souplesse et une maîtrise des mesures il est fondamental de pouvoir accéder facilement aux capteurs de pressions; d'où la nécessité des piézomètres.

Ces piézomètres seront crépinés sur une certaine hauteur, leur diamètre doit être assez grand pour permettre de passer aisément le capteur et son habillage éventuel. Le crépinage de ces piézomètres doit être spécialement étudié afin d'éviter toute perte de charge qui fausserait la mesure. Ce crépinage est à définir en pourcentage (pourcentage de la surface de vides par rapport à la surface du piézomètre) et en forme et dimension des crépines.

En tout cas, le crépinage doit être le plus important possible compte-tenu de la résistance mécanique des tubes.

L'emplacement des capteurs dans le noyau pourrait être comme suit :

- une série de capteurs sera placée horizontalement pour évaluer l'amortissement de la pression en fonction de l'avancement dans le noyau;

- deux capteurs, au moins, doivent être placés à la même verticale pour vérifier si la répartition de la pression est hydrostatique ou pas.

- les capteurs doivent être calés à l'intérieur des piézomètres.

Deux solutions sont possibles pour le calage des capteurs et l'adoption d'une hauteur de crépinage, elles sont schématisées ci-contre.

- dans la première solution le capteur est calé à l'aide d'une perche rigide, le crépinage dans ce cas peut dépasser le niveau de l'eau;

- dans la deuxième solution le capteur est calé par du gravier pius un coulis de ciment qui empêche tout mouvement ascendant.

La première solution a l'avantage de la facilité de récupération du capteur et d'accès au piézomètre pour son entretien.
La seconde permet d'éviter l'écoulement retardé qui risque d'arriver dans le piézomètre par rapport à l'écoulement dans le noyau de la digue; cependant, un crépinage important limitera la portée de ce phénomène.

6) Enfin, ci-après des remarques sur les capteurs pour enregistrer des pressions rapidement variables en fonction du temps.

La période des fluctuations étant de l'ordre d'une dizaine de secondes, les capteurs doivent avoir un temps de réponse assez faible pour enregistrer de telles variations; soit un temps de réponse de l'ordre de la seconde. Cependant, il y a une condition plus restrictive en ce qui concerne la rapidité de réponse des capteurs; il s'agit du déphasage éventuel, certainement très faible, qui existe entre les pressions en deux points du noyau. Ainsi, un temps de réponse de l'ordre du $1 / 10^{\circ}$ de seconde semble être souhaitable.

L'amplitude des fluctuations est de l'ordre de grandeur de la houle incidente à l'amortissement du noyau près. Soit des pressions très inférieures à $10 \mathrm{~m}$ d'eau (1 bar). Toutefois, le risque de développement de pressions interstitielles exceptionnelles par temps de tempête oblige à prendre une plus large bande de mesure. Les capteurs devront donc être sensibles à des pressions de 0 à 3 bars environ.
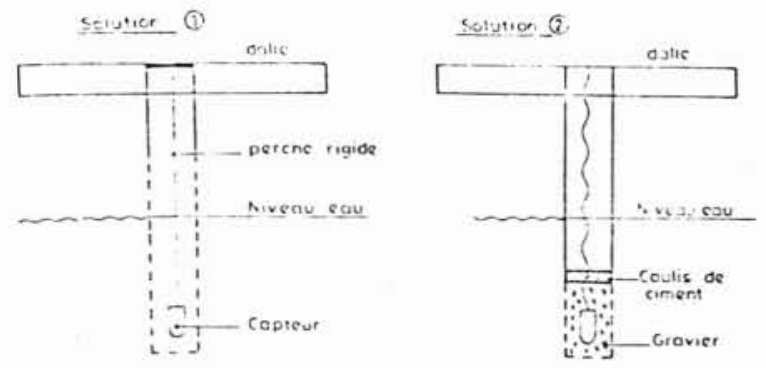

Figure 2 